\title{
Endoscopic removal of gallstone impacted in the rectum
}

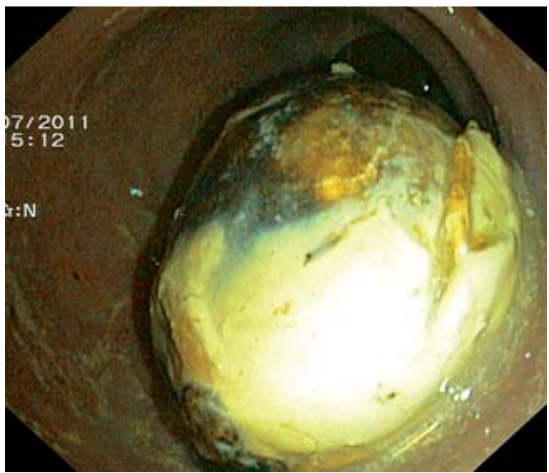

Fig. 1 Endoscopic image of the peach pit-like foreign body impacted in the rectum.

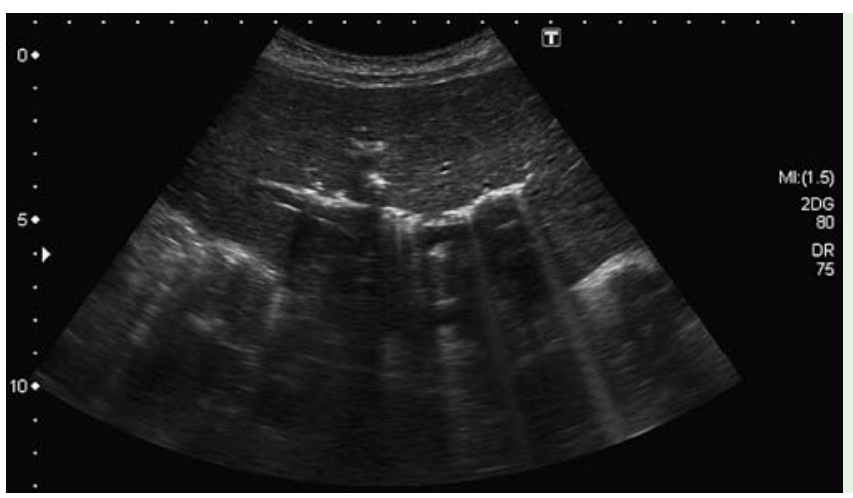

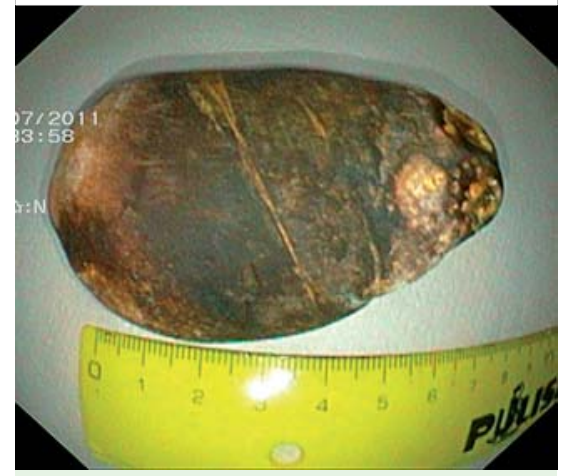

Fig. 2 The gallstone which was extracted from the rectum.

Fig. 3 Ultrasound image showing pneumobilia.
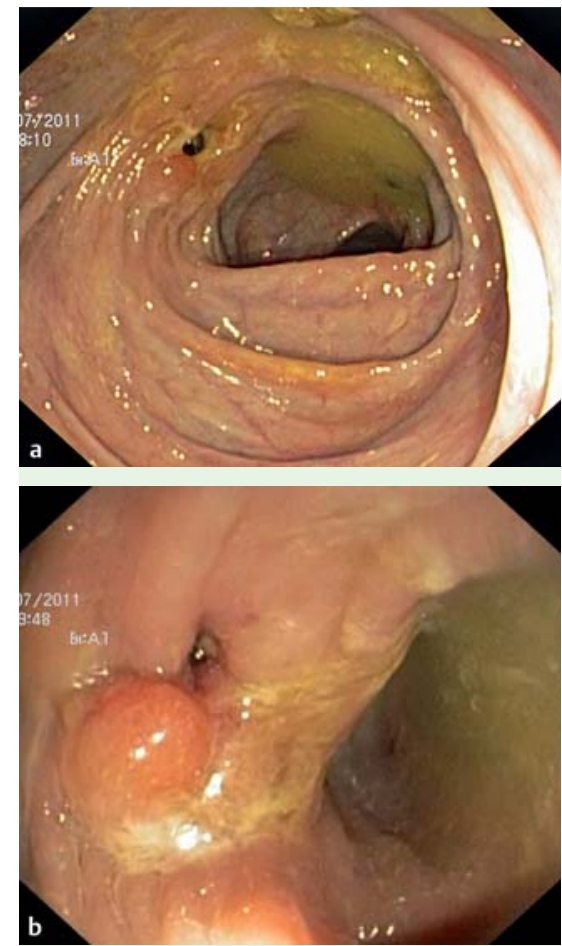

Fig. 4 Fistulous orifice at the hepatic flexure of the colon. No bile drainage was noted from the fistulous orifice.

After gallstone removal, CEFs can be repaired in a one-stage surgical procedure along with cholecystectomy. However, in elderly patients at high surgical risk, CEFs may be treated conservatively even if the risk of recurrent biliary ileus remains elevated.

Endoscopy_UCTN_Code_TTT_1AQ_2AH

\section{Competing interests: None}

\section{F. Perri, F. Terracciano, M. Gentile,} A. Merla, D. Scimeca, A. Andriulli Endoscopic Unit, IRCCS Casa Sollievo della Sofferenza Hospital, San Giovanni Rotondo, Italy 


\section{References}

1 Balthazar EJ, Gurkin S. Cholecystoenteric fistulas: significance and radiographic diagnosis. Am J Gastroenterol 1976; 65: 168-173

2 Abou-Saif A, Al-Kawas FH. Complications of gallstone disease: Mirizzi syndrome, cholecystocholedochal fistula, and gallstone ileus. Am J Gastroenterol 2002; 97: 249-254

3 Hinchey PR. Gallstone ileus. Arch Surg 1943; 46: $9-49$
4 Milsom JW, MacKeigan JM. Gallstone obstruction of the colon. Report of two cases and review of management. Dis Colon Rectum 1985; 28: 367-370

5 Skulstad S, Olsen L, Rokke O. Intestinal obstruction by a large gallstone in the rectum: successful catheter-aided extraction. Eur J Surg 2000; 166: $422-425$

\section{Bibliography}

DOI $10.1055 / \mathrm{s}-0030-1256949$

Endoscopy 2011; 43: E408 -E409

(c) Georg Thieme Verlag KG Stuttgart · New York . ISSN 0013-726X

\section{Corresponding author}

\section{F. Perri, MD, PhD}

Endoscopic Unit

IRCCS Casa Sollievo della Sofferenza Hospital 71013 San Giovanni Rotondo

Italy

Fax: +39-882-410989

fp.perri@libero.it 